oil remains supercooled for a long time, thus increasing unduly the time required to expel the dissolved gas.

While these experiments were nearing completion, it came to our attention that a freeze-thaw technique somewhat similar to this had been used by research workers $^{6,7}$ investigating the spin lattice relaxation times of protons in organic liquids. In these investigations, the main purpose had been to expel the dissolved oxygen from the liquids, as the presence of this gas vitiated the results. The usefulness of this technique in outgassing manometric liquids does not seem to have been recognized so far.

Finally, it is instructive to examine here the physical processes underlying the freeze-thaw technique as evidenced by experiments. As the manometric tube is cooled suddenly, solidification of the oil starts at the inner surface of the tube. However, as the crystalline phase grows, the dissolved gases are believed to be expelled from the frozen state. This results in an increase in the quantity of dissolved gas in the liquid phase near the phase boundary until it gets saturated. Any further expulsion of gas from the growing solid phase is accompanied by the formation of the gaseous phase, the interface with the solid appearing with a cracking sound. The gas thus trapped in the cracks is released during the thawing process.

The air dissolved in the oil also appears to play an important part in determining the time taken by the oil to freeze in tubes of large diameters. For instance, in a tube $3.18 \mathrm{~cm}$ diam filled with oil fully saturated with air, the freezing time is a minimum, being about 15 min when immersed in liquid nitrogen. After expelling the dissolved air the first time, the time required to freeze is found to increase to about $1 \mathrm{~h}$, and after the second expulsion of air, the oil remained immersed in liquid nitrogen for over $3 \mathrm{~h}$ without freezing. This indicates that the thermal conductivity of the oil is perhaps influenced by the dissolved air and becomes very small as the gas gets completely expelled. It also suggests that in order to determine the true conductivity of a viscous liquid, it is necessary to outgas it completely before carrying out measurements.

The authors wish to acknowledge the help rendered by E. A. Branley, M. Iovine, and C. N. Miceli in setting up the vacuum system and the recording device. Their thanks are also due to the authorities of L.S.U.N.O., Dr. B. J. Good and Dr. L. D. Dureau in particular, for giving them the opportunity and the facilities to work in the Physics Department of the University.

${ }^{1}$ M. A. Biondi, Rev. Sci. Instr. 24, 989 (1953).

2 R. L. Phipps and J. H. Bloom, Vacuum 11, 35 (1961).

3 J. V. McAllan, J. Sci. Instr. 42, 290 (1965).

${ }^{4}$ A. T. J. Hayward, J. Sci. Instr. 40, 173 (1963).

${ }^{5}$ R. Hoimes and G. R. Jones, Vacuum 13, 231 (1963).

${ }^{B}$ A. W. Nolle and P. P. Mahendroo, J. Chem. Phys. 33, 863 (1960).

${ }^{7}$ G. W. Nederbragt and C. A. Reilly, J. Chem. Phys. 24, 1110 (1956).

\section{FET Operational Amplifiers as Fast Electrometers*}

\author{
W. H. Wing and T. M. Sanders, JR. \\ H. M. Randall Laboratory, University of Michigan, \\ Ann Arbor, Michigan 48104
}

(Received 15 May 1967)

$I^{T}$

$\mathrm{T}$ is our purpose in this note to acquaint experimentalists who may be unfamiliar with these devices with the usefulness of operational amplifiers using field-effect transistors in the input stage in experiments requiring detection of weak transient currents. We have used these amplifiers in measuring charged particle currents in liquid helium and in detecting weak modulated photocurrents, and they appear to have a wide range of possible applications. The electrometers we have built utilize a relatively inexpensive commercial operational amplifier and a minimal amount of other circuitry. Typical performance obtained is minimum detectable current $5 \mathrm{pA}$, with a rise time of 10 to $20 \mu \mathrm{sec}$; faster response can be obtained with some sacrifice of sensitivity.

The basic circuit used is the familiar current source amplifier shown in Fig. 1. Input capacitance, including the effect of cables, is represented by $C$; capacitance across the feedback resistor, either intentional or parasitic, is designated as $C_{\mathrm{F}}$. It is quite simple to write down the transfer function for this circuit, including the effects of the $6 \mathrm{~dB}$ per octave rolloff designed into the operational amplifier. The conclusion is that if $C_{\mathrm{F}}$ is too small the circuit will be underdamped; if it is too large the circuit will be sluggish and will tend to integrate pulsed currents. Critical damping is achieved when $C_{F}$ satisfies the equation

$$
R C_{\mathrm{F}}=2\left(R C / 2 \pi \nu_{0}\right)^{\frac{1}{2}},
$$

where $\nu_{0}$ denotes the frequency at which the amplifier open-loop gain is unity. At critical damping ${ }^{1}$ the circuit rise time is

$$
\tau=\frac{1}{2} R C_{F}=\left(R C / 2 \pi \nu_{0}\right)^{\frac{1}{2}} .
$$

Best current sensitivity is obtained by raising $R$ until the noise is dominated by the input current noise of the ampli-

Fig. 1. Block diagram of the electrometer amplifier. C represents input and cable capacitance, $R$ is the feedback resistor, and $C_{F}$ any shunt capacitance.

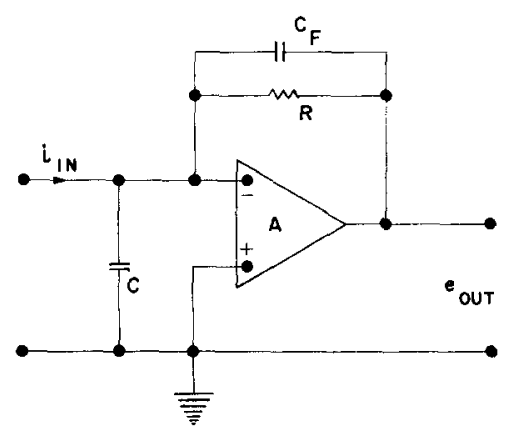


fier. Further increase in $R$ results in degradation of circuit rise time without any further reduction in midband noise current.

The electrometers we have constructed are built around the Philbrick model P25AH operational amplifier whose nominal properties are $\nu_{0}=30 \mathrm{MHz}$, and input current noise $\cong 3 \mathrm{pA}$ rms. The optimum value of $R$, according to the considerations of the last paragraph, is in the range $10^{8}$ to $10^{9} \Omega$. For $R=10^{9} \Omega, C=100 \mathrm{pF}$. Equation (1) yields the value $C_{\mathrm{F}}=0.05 \mathrm{pF}$ for critical damping. It is possible to approach critical damping, using some care in layout and construction, and it is important to choose for $R$ a resistor with suitably low shunt capacitance. We have found Victoreen Hi-Meg resistors to be quite suitable. The amplifier socket is mounted on a chassis which is divided into input and output sections by a grounded partition, which is connected to an unused pin on the amplifier socket. The input connector and the amplifier input terminals are on one side of the partition; the output connector, the amplifier output terminal, and power supply leads are on the other. The feedback resistor $R$ passes through a small hole in the partition. If values of $R$ less than $10^{9} \Omega$ are used, to achieve shorter rise times, it is necessary to add some capacitance across $R$.

Best performance obtained with this device, in applications with 10 to $100 \mathrm{pF}$ cable capacitance at the input, is rms current noise, $2 \mathrm{pA}$; rise time, $10 \mu \mathrm{sec}$. We have noted variations in input noise of about a factor of three in different examples of the P25AH amplifier.

* This work was supported in part by the U. S. Atomic Energy Commission.

${ }^{1}$ In writing Eqs. (1) and (2) we have neglected some small terms. Critical damping cannot be reached if $C$ is too large.

\section{Ultrasonic Interferometer-Laser Optical Dif- fraction Cell for Rapid Determination of the Velocity of Sound in Liquids}

\author{
M. E. Pedinoff and H. SegurN* \\ Hughes Research Laboratories, Malibu, California 90265
}

(Received 11 April 1967; and in final form, 22 May 1967)

$\mathrm{T}$ HE use of optical diffraction spectra produced by ultrasonic waves for the determination of sound velocity in liquids is well known. ${ }^{1-4}$ Prior to the development of the coherent gaseous laser, it was necessary to employ rather complicated optical systems to generate the collimated light beams used to produce the diffraction spectra and to resolve the diffraction orders. In this note, we describe a liquid ultrasonic resonator which has optical transmission windows attached to its side walls for generating laser beam diffraction spectra. The device can be used to measure the velocity of sound to within $1 \%$ accuracy, provided the radio frequency of the oscillator is known to better than $1 \%$. The device is easily constructed and simple to operate, and produces useful data rapidly.

The phenomenon of light diffraction by ultrasonic waves in liquids has been described by Debye and Sears, ${ }^{1}$ Brillouin, ${ }^{2}$ Raman, ${ }^{3}$ and Willard ${ }^{4}$; the observed spectra fall into either the Raman-Nath regime or the Bragg scattering regime. The equation governing the location of the diffraction orders for the former is given by

$$
\left(\sin \theta_{\mathrm{m}}-\sin \theta_{1}\right)=n \lambda / \Lambda,
$$

and the concomitant Doppler frequency shifts in these orders for traveling ultrasonic waves are given from the work of Raman and Nath ${ }^{3}$ by

$$
\nu_{\mathrm{m}}=v_{0}+n \nu_{\mathrm{s}},
$$

where $\lambda$ is the wavelength of light in the diffracting medium, $\Lambda=v_{\mathrm{s}} / \nu_{\mathrm{s}}$ is the wavelength of sound in the medium, $v_{\mathrm{s}}$ is the velocity of sound, $\nu_{\mathrm{s}}$ is the frequency of the sound wave, $n$ is the order of the interference $(0, \pm 1, \pm 2, \cdots), \theta_{1}$ is the angle of incidence of the light beam on the diffracting medium, and $\theta_{\mathrm{m}}$ is the angle of diffraction of the light beam from the diffracting medium. Cummins et al..$^{5}$ experimentally verified the frequency shifts predicted in Eq. (2), as well as the single sideband nature of the modulation. Willard ${ }^{4}$ has shown that when an ultrasonic transducer of large dimension is used, so that the light beam intersects two or more successive ultrasonic maxima (or minima), most of the diffracted energy appears in the lower orders. If the orientation of the ultrasonic transducer is adjusted so that the incident beam and one diffracted beam are equally inclined relative to the face of the transducer, a strong single frequency-shifted diffraction maximum results. This phenomenon is referred to as Bragg scattering of light waves by an ultrasonic optical grating. In the case of Bragg scattering, the equations are given by

$$
\sin (\theta / 2)=n \lambda / 2 \Lambda
$$

for the diffraction maxima, and by Eq. (2) for the frequency shifts, where $\theta=2 \theta_{1}=-2 \theta_{\mathrm{m}}$ is the angle between the incident and the diffraction beam and the beams are symmetrically inclined with the transducer face. If the medium contains two opposite collinear traveling ultrasonic waves which form a standing wave, two optical beams of opposite frequency shift will exist at each diffraction maximum position. This can be easily seen from Eq. (2) by allowing $n$ to take on the values +1 and -1 corresponding to the oppositely directed ultrasonic waves. Two light beams are obtained, of frequency $\nu_{0}+\nu_{s}$ and $\nu_{0}-\nu_{s}$, which yield a carrier beam at frequency $\nu_{0}$ modulated at frequency $\nu_{\mathrm{s}}$. For values of $\nu_{\mathrm{s}}$ lying in the megacycle region, the modulation must be detected by a fast photodetector; however, the eye can detect the presence of the diffracted beam since it counts optical quanta, or acts as a square 